\title{
Dynamic calibration for improving the speed of a parallel-aligned liquid-crystal-on-silicon display
}

\author{
Joaquín Otón, ${ }^{1}$ Pierre Ambs, ${ }^{2}$ María S. Millán, ${ }^{1, \star}$ and Elisabet Pérez-Cabré ${ }^{1}$ \\ ${ }^{1}$ Department of Optics and Optometry, Technical University of Catalonia, Terrassa, Barcelona, Spain \\ ${ }^{2}$ Laboratoire Modélisation Intelligence Processus Systèmes, École Nationale Supérieure d'Ingénieurs \\ Sud Alsace-Mulhouse, Université de Haute Alsace, Mulhouse Cedex, France \\ ${ }^{*}$ Corresponding author: millan@oo.upc.edu
}

Received 3 April 2009; revised 22 July 2009; accepted 24 July 2009;

posted 27 July 2009 (Doc. ID 109654); published 4 August 2009

\begin{abstract}
The speed of most parallel-aligned liquid-crystal-on-silicon (LCoS) spatial light modulators (SLMs) is limited to the video rate of their drivers, which is a limitation for high-speed applications. However, the LCoS SLM presented here has a driver allowing a frequency range of up to $1011 \mathrm{~Hz}$. Using the static phase modulation characterization and the static lookup table (LUT), the phase modulation characterization versus frequency shows that the SLM can operate at around $130 \mathrm{~Hz}$ or even higher for small phase changes and at $32 \mathrm{~Hz}$ for extreme phase changes. A dynamic calibration is carried out, and we propose a method allowing an increase of the frame rate while maintaining a maximum phase modulation of $2 \pi$. Experimental results of dynamic diffractive optical elements displayed on the SLM at a frame rate of $205 \mathrm{~Hz}$ show that the dynamic LUT improves the reconstruction quality. (C) 2009 Optical Society of America

OCIS codes: $\quad 220.1000,050.1970,230.6120,230.3720$.
\end{abstract}

\section{Introduction}

Numerous papers on spatial light modulators (SLMs) have been published in recent years as a consequence of the large availability of highperformance devices in terms of modulation capability, resolution, and speed. Among the SLMs that are commercially available, the reflective type of pure phase SLMs, based on liquid-crystal-on-silicon (LCoS) technology [1-3] are particularly interesting for applications requiring a high optical efficiency, such as dynamic diffractive optical elements (DOEs).

Recent papers have reported different applications of LCoS SLMs, such as pulse shaping [4], hologram reconstruction [5], computer generate $\bar{d}$ holograms [6], DOEs [7], optical tweezers [8], and optical metrology [9]. A large number of contributions in the literature over the last decades account for the importance of the characterization and the aberration compensa-

0003-6935/09/234616-09\$15.00/0

() 2009 Optical Society of America tion techniques of LCoS SLMs [10-20]. A good and complete characterization is an essential step in SLM initialization, particularly if the SLM is to be used in applications that require high wavefront control. The quality of the obtained wavefront after passing through the SLM strongly depends on the knowledge of the device response. Usually, the signal must be modified before addressing it to the SLM to compensate for the distortions internally introduced by the device and, hence, to eventually reproduce the desired wavefront. Among the published research work, much more attention has been paid to the static calibration of SLMs, and very few studies appear in the literature dealing with their dynamic behavior. Although the calibration of a SLM for steady signals is a necessary first step in device initialization, it is also important to know the SLM performance for a time-varying signal, particularly because many of the interesting properties of SLMs are related to their potential capability of being programmable and dynamically adaptive. 
Since SLMs are mainly produced for video displays, their refresh rate up to video frequency is a usual limit for their maximum frame rate. However, speed is an important feature of LCoS SLMs for a number of applications that require dynamic control of the wavefront, such as in real-time wavefront correction, adaptive aberration compensation, beam steering, beam shaping, active diffractive optics, dynamic holographic optical trapping systems, turbulence-emulating systems, and optical signal processing. For these applications, higher SLM operating speeds are desired.

Recently, Serati from Boulder Nonlinear Systems (BNS) presented a high-resolution phase-only SLM with submillisecond response [21] using dualfrequency nematic liquid crystal $(\overline{\mathrm{LC}})$.

So far, to the best of the authors' knowledge, temporal calibration of the SLM response has not been considered as widely as the static calibration. The spatiotemporal transfer behavior of the device is analyzed in Ref. [17] to control the laser radiation at short pulse duration. They measured the dispersion effects (pulse broadening or compression) introduced by the SLM for impinging subfemtosecond laser pulses. Some studies of the dynamic response of ferroelectric binary SLMs have been reported by BNS $[22,23]$. A decade ago, Cho et al. [24] reported on the temporal characteristics of a BNS zero-twist nematic SLM operating in reflective mode, with eight equally spaced phase levels between 0 and $2 \pi$. The reproduction of alternated phase images of uniform pixel values with increasing temporal frequency showed a quality strongly dependent on the phase difference existing between the alternated images, limiting arbitrary phase modulation speed to approximately $4.5 \mathrm{~Hz}$. Regarding 8 bit phase modulation, BNS has also analyzed the capabilities of new electrically controlled birefringence (ECB) SLMs to overcome the limit established by the video frame rate addressing operation and to effectively form static phase patterns with these devices [25]. Very recently, Lizana et al. [26] have demonstrated that, for a twisted nematic $\overline{\mathrm{LC}} \mathrm{oS}$ display, the fluctuations of the LC molecular orientation due to the way the electric signal is addressed to the display, produces fluctuations of the state of polarization of the reflected beam, causing a depolarization effect. In Ref. [27], the authors proposed a phase-only diffraction grating method, taking time-resolved measurements of the intensity of the generated diffraction orders, to experimentally detect the time fluctuation of the phase modulation of the twisted nematic LCoS SLM.

In this paper, a dynamic characterization of an LCoS SLM is presented. From the experiments carried out in this work, we aim to know the experimental constraints that influence the capacity of such a device to dynamically generate a varying phase pattern. We want to establish the best working conditions to assure that the signal to be reproduced in the SLM is not significantly distorted by the frame rate used to display a dynamic phase distribution.
The remainder of this paper is organized as follows. In Section 2, we briefly describe the static calibration of the SLM. Speed characterization is detailed in Section $\underline{3}$ and a dynamic calibration to overcome the frequency limitation is proposed in Section 4. Experimental results are provided in Section $\overline{5}$, and finally, conclusions are summarized in Section $\underline{6}$.

\section{Principle of the Spatial Light Modulator Characterization}

\section{A. Technical Characteristics}

This paper is related to the P256 zero-twist nematic liquid-crystal (ZTN-LC) model manufactured by BNS in 2003. This SLM is based on the zero-twist or parallel-aligned LCoS technology and works as a reflective display, in the pure phase-modulation regime. The device is a $256 \times 256$ pixel array of $4.608 \mathrm{~mm} \times 4.608 \mathrm{~mm}$ size, with $18 \mu \mathrm{m}$ pixel pitch, $85 \%$ fill factor, $65 \%$ zero-order diffraction efficiency and 8 bit electrically addressable levels. Other features of this modulator are specified in Table 2 of Ref. [19]. Regarding the dynamic characteristics of the display, it has a frame rate frequency of up to $150 \mathrm{~Hz}$, according to technical specifications. However, the driver allows a frequency range of up to $1011 \mathrm{~Hz}$.

\section{B. Phase Calibration}

All experimental data presented in this paper were obtained using a He-Ne laser at $633 \mathrm{~nm}$ wavelength.

The principle of the phase calibration of the BNS SLM was presented in detail in a previous paper [19]. We used the method proposed by Zhang et al. [28] to calibrate the SLM. It is based on displaying binary phase (Ronchi) gratings and measuring the intensities in the diffraction pattern at the Fourier plane (Fig. 1). A diffraction binary grating of square fringes with gray-level values given by the reference gray level $N_{\text {ref }}$ and the varying gray level $N_{v}$ is addressed to be displayed on the SLM. Let us denote

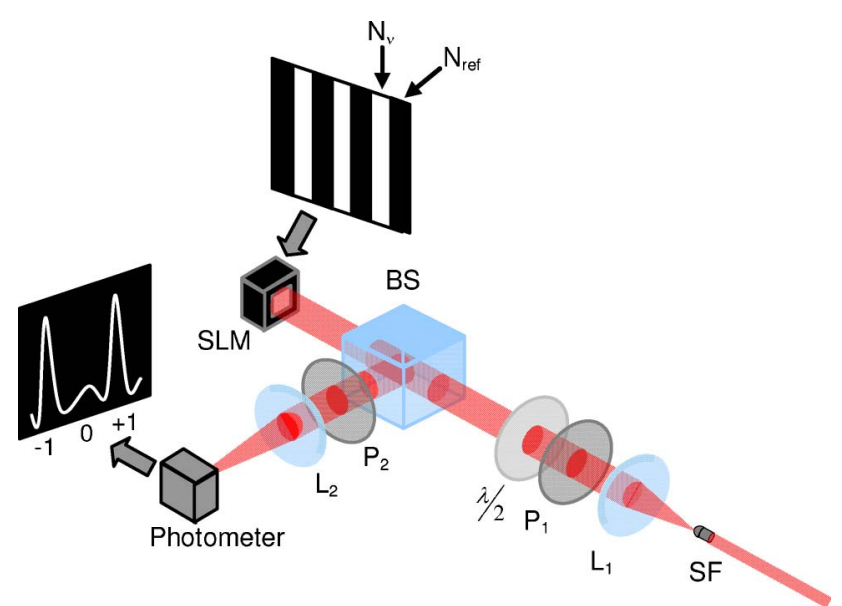

Fig. 1. (Color online) Setup for the calibration of the BNS SLM using the Ronchi grating method proposed by Zhang [28]. The gray level values of the grid are $N_{v}$ and $N_{\text {ref }}$. 
$\Delta \phi(N)=\phi\left(N_{v}\right)-\phi\left(N_{\text {ref }}\right)$ the phase shift corresponding to the gray levels $N_{v}$ and $N_{\text {ref }}$. It is assumed that the coupled amplitude modulation is negligible as it corresponds to a pure phase grating. The intensity at the first diffraction order is proportional to

$$
I_{1}(\Delta \phi) \propto[1-\cos \Delta \phi(N)] .
$$

The intensity function of Eq. (1) shows a maximum value for phase shifts that are multiples of $\pi$ and a minimum value for those phase shifts that is a multiple of $2 \pi$. The reference gray level was set to $N_{\text {ref }}=$ 128, which was equivalent to the OFF state. The experimental results obtained for the intensity of the first diffraction order $I_{1}(\Delta \phi)$ are plotted in Fig. 2(a). This plot corresponds to the phase response of the BNS SLM on average on the device aperture. The maximum peak values can be associated with aver-
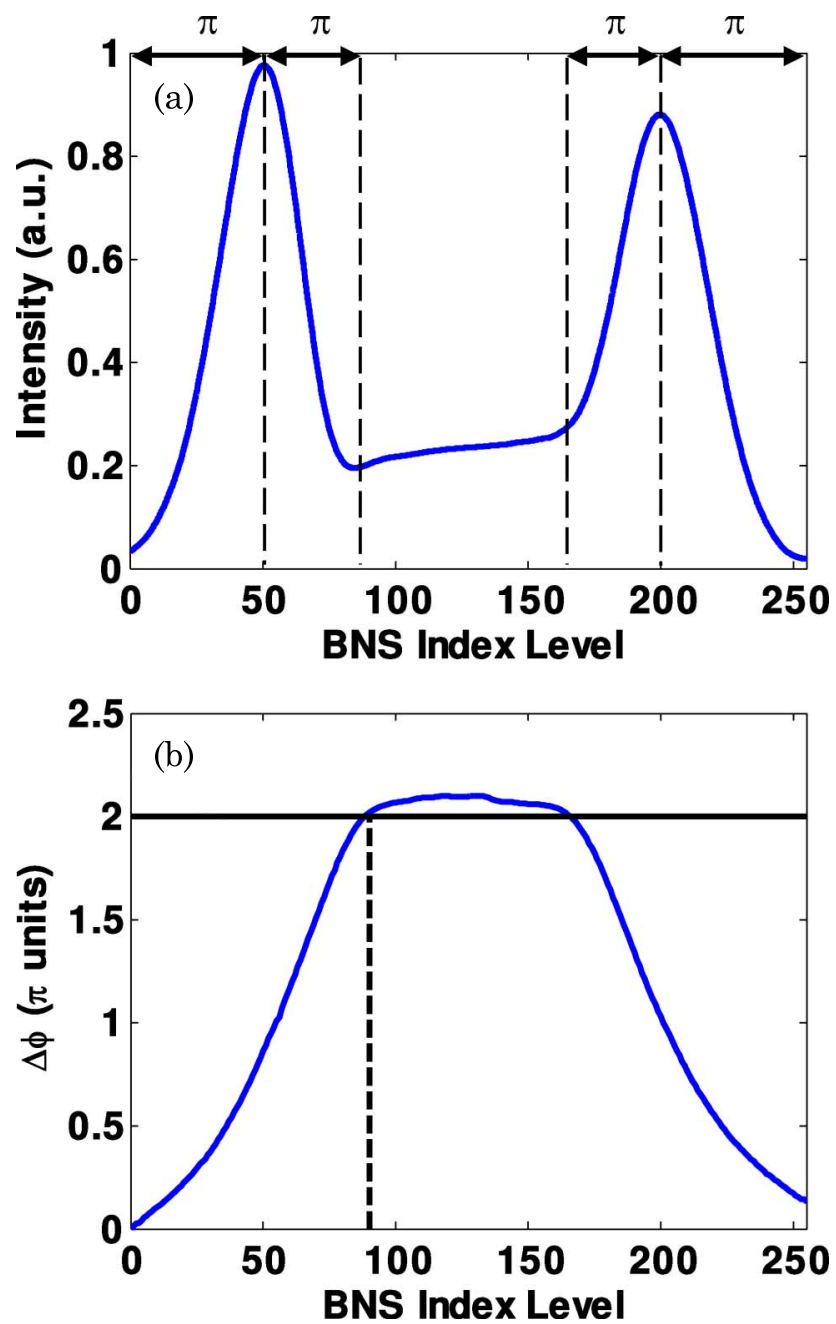

Fig. 2. (Color online) Experimental measurements of the phase shift generated by the nematic LC SLM for different BNS index levels when no LUT is applied. (a) Intensity of the first diffraction order ( $Y$ axis) when a Ronchi grating with variable BNS index levels from 0 to 255 ( $X$ axis) is displayed on the SLM. (b) Phase shift computed from the data of curve (a). A phase modulation range of $2 \pi$ is used to fix a BNS index level range of $[0,87]$ and from it, to generate a LUT. age phase shifts of $\Delta \phi(N)=\pi$. However, it does not imply that phase shifts of $\pi$ are uniformly generated by the Ronchi phase grating actually displayed on the SLM aperture. It has been proved that this sort of device can be spatially nonuniform $[16,19]$ and, consequently, although the phase grating addressed to the device is binary, the phase grating actually displayed has different phase shifts depending on the zone of the aperture. It can be seen that the LC device performs nearly symmetrically around the gray level $N_{v}=128$. Since the nematic LC does not respond to the polarity of the electric field, complementary pixel values $N_{v}$ and $255-N_{v}$ result in the same phase shift. On both sides of each maximum value, we take two low values to fix the range limits $\Delta \phi(N)=\{0,2 \pi\} \quad$ [Fig. 2(a)]. From Fig. 2(a) and Eq. (1) we plot the phase shift versus the BNS index level in Fig. 2(b), where it can be seen that the phasemodulation range slightly exceeds $2 \pi$. It should be noted that these measurements were conducted 18 months after the measurements presented in our previous paper [19], and that the phase-modulation characteristics and the maximum phase shift changed during this period of time. This fact can explain the differences in the experimental data.

In many applications, the phase is computed modulo- $2 \pi$. For this reason, and taking into account the calibration of Fig. 2(b), which correspond to the static display regime, we tentatively limited the BNS index level range to $[0,87]$. To linearize the phase response, we built a lookup table (LUT). For practical reasons, this LUT extended the BNS index level range [0,87] into a more common 8 bit range of mapped gray levels $[0,255]$. With this LUT, a phase shift of exactly $2 \pi$ is achieved when the input pixel value varies between 0 and 255 .

Following the method described in Ref. [19], we used a Michelson interferometer to test the phase shift for different regions of the device aperture. We divided the SLM into different regions and, in each region, we measured the value of the BNS index level of the static LUT that corresponds to a $2 \pi$ phase shift, as shown in Fig. 3. From these values, we computed a specific LUT for each region. The use of this multiLUT minimizes the phase discontinuities in the phase pattern generated by the SLM [19].

According to BNS, the LCoS SLMs have an inherent curvature causing a wavefront distortion of the reflected wave [15]. The silicon backplane is one of the largest contributors to the curvature of an SLM, but the LC also contributes to the reflected wavefront distortion. We used a Michelson interferometer to measure the reflected wavefront distortion of the SLM. The mirror is tilted and the resulting fringe pattern is analyzed to compute the wavefront [Figs. 4(a) and 4(b)]. Knowing the Zernike coefficients of the distorted wavefront, the wavefront that must be added to the SLM to compensate the distortions can be computed. In our experimental conditions, the results obtained after applying the compensation are represented in Figs. $4(\mathrm{c})$ and $4(\mathrm{~d})$. 


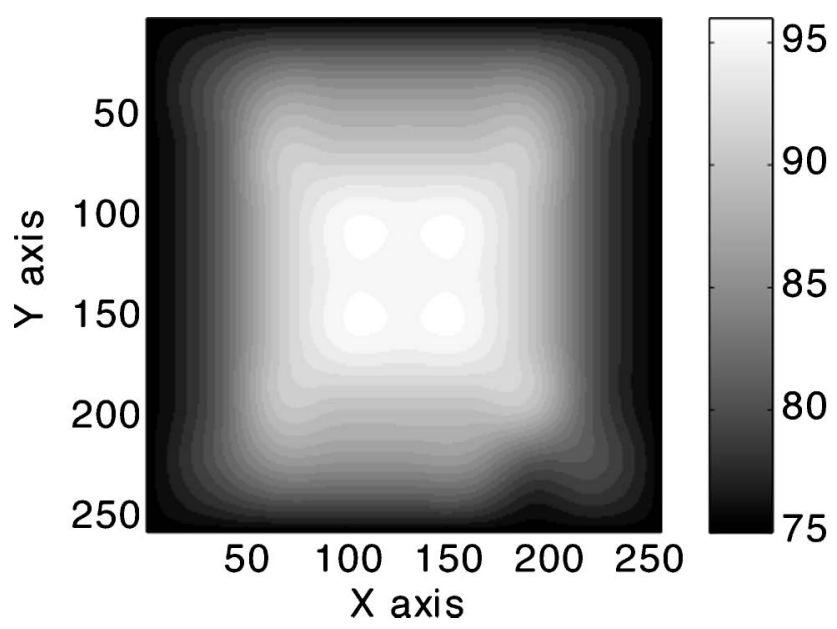

Fig. 3. Implementation of a multi-LUT. Gray-level value giving a $2 \pi$ phase shift for the different regions of the SLM.

Although the phase modulation range of the modulator has decreased, the compensation shown in Figs. 4(c) and 4(d) is still good (see Fig. 11 of Ref. [19] for comparison).

\section{Speed Characterization}

The driver of the BNS SLM allows a frequency range of up to $1011 \mathrm{~Hz}$ and this is one of its advantages over other phase LC SLMs that are limited to the video rate. According to the technical specifications of the LCoS SLM, the maximum usable frame rate ranges from 25 to $150 \mathrm{~Hz}$, depending on phase stroke, wavelength, and temperature.

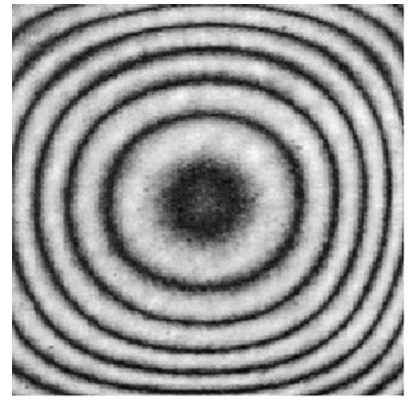

(a)

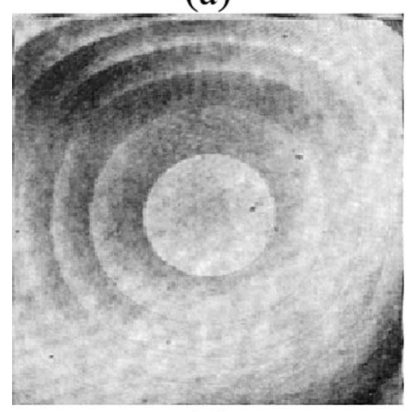

(c)

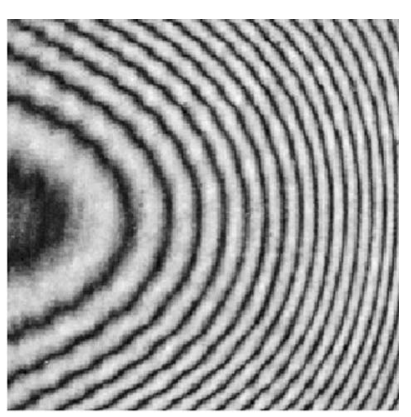

(b)

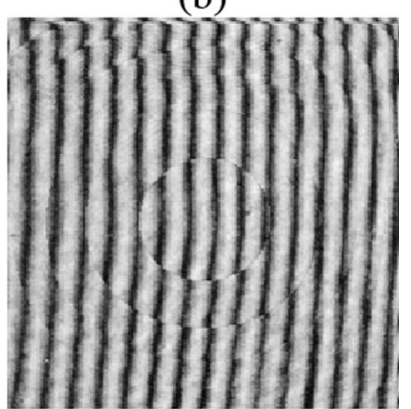

(d)
Fig. 4. Interferogram of the modulator: (a) parallel reference mirror, without compensation of wavefront distortion; (b) tilted reference mirror, without compensation; (c) parallel mirror, with compensation of wavefront distortion; and (d) tilted mirror, with compensation.
In the first experiment, we studied the dynamic response of the SLM for small phase increments. For this, we considered again the SLM in the setup of Fig. 1. To analyze the dynamic behavior of the SLM, we used the LUT defined Section 2 and measured experimentally the first diffraction order $I_{1}(\Delta \phi)$ that showed the sinusoidal appearance [Fig. 5(b)]. Two phase Ronchi gratings that differ by a small phase variation between them were alternately addressed, for a time $t$ each, to the SLM with increasing frequency, from 1 to $1011 \mathrm{~Hz}$. The phase distributions of the binary gratings were defined by $\left[N_{\text {ref }}=128\right.$, $\left.N_{v}=n\right]$ and $\left[N_{\text {ref }}=128, N_{v}=n+1\right]$, with the graylevel value varying from $n=0$ to $n=255$ [Fig. 5(a)]. Figures 5(b) and 5(c) show the experimental results. It can be seen that, even at $1011 \mathrm{~Hz}$, the maximum phase shift reaches $2 \pi$, although, for frequencies higher than $126 \mathrm{~Hz}$, the SLM does not stabilize between two consecutive levels.

In the second experiment, we analyzed the SLM speed to the maximum transition between gray levels, i.e., between the levels 0 and 255 . To cover the gap between the extreme levels 0 and 255, the molecules of the LC have to cross all the intermediate levels. In this experiment, the two Ronchi phase gratings, addressed alternately for a time $t$ each, correspond to $\left[N_{\text {ref }}=128, N_{v}=0\right]$ and $\left[N_{\text {ref }}=128, N_{v}=\right.$ $255]$. Both gratings are theoretically equivalent and, consequently, they should give the same result for the intensity of the first diffraction order, which corresponds to a maximum peak value. But this is only true at very low frame rates, for which a T-shaped intensity plot is obtained (Fig. $\underline{6}$ ). In Fig. $\underline{6}$, the intensity of the first diffraction order (in arbitrary units) is presented versus time (in $t$ units). The frame rate (in hertz) is equal to $1 / t$, with $t$ in seconds. As the frame rate increases, the behavior of the SLM is more and more distant from the expected response. From the experimental results shown in Figs. 6(a)-6(f), we con-

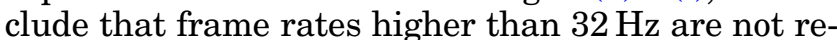
liable for displaying time variant phase distributions that involve the maximum 0-255 gray-level variation ( $0-2 \pi$ phase transition) addressed to the same pixel (LC molecules).

\section{Dynamic Calibration}

In this section, we present a method to overcome, in certain limits, the previous frequency limitation. The idea is to implement a dynamic LUT, taking into account a range of BNS index level values more extended than the range fixed during the static calibration for increasing the frequency while maintaining a maximum phase modulation of $2 \pi$.

We used again the setup of Fig. 1 and the method based on displaying different binary phase gratings alternately at increasing frequency. Figure 2(b) shows the phase modulation used to generate the static LUT for a BNS index level range of $[0,87]$. In Fig. 5 , the phase was only varying by a small increment, but here the purpose is to evaluate the ability to achieve any phase modulation when the SLM 


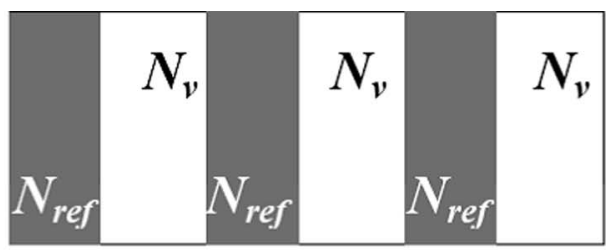

$$
\left(N_{\text {ref }}=128, N_{v}=n\right) \longrightarrow\left(N_{\text {ref }}=128, N_{v}=n+1\right)
$$
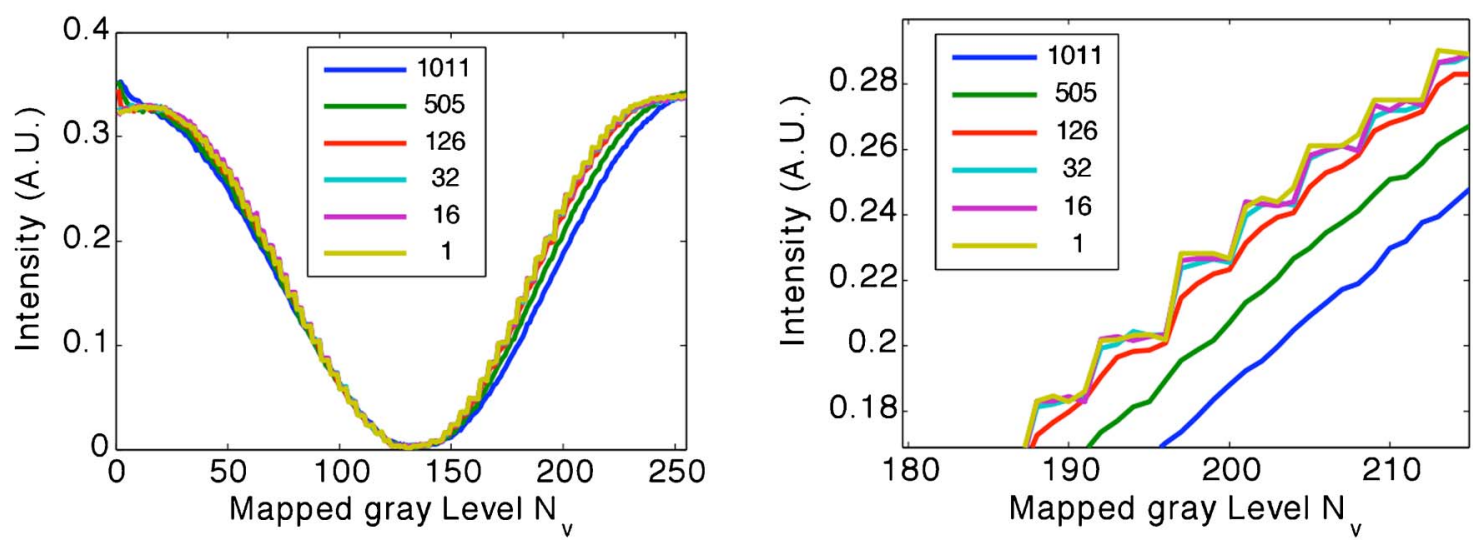

Fig. 5. (Color online) Intensity curves of the first diffraction order for a binary phase grating that changes a small increment with increasing frame rate.

starts from a given reference. Thus, the two phase gratings that are to be alternately addressed to the display are generated according the static LUT with the values $\left[N_{\text {ref }}=N_{\pi}, N_{v}=N_{0}\right]$ and $\left[N_{\text {ref }}=\right.$ $N_{\pi}, N_{v}=n$ ] with $N_{0}=0$ and $n=\{0 \ldots 255\}$ [Fig. 7(a)]. In each period of time $T$, the reference phase grating $\left[N_{\text {ref }}=N_{\pi}, N_{v}=N_{0}\right]$ was displayed for a time $t_{r}=$ $3 T / 4$, while the other phase grating $\left[N_{\text {ref }}=N_{\pi}, N_{v}=\right.$ $n]$ was displayed just for a time $t=T / 4$. Operating in this way, the molecules are forced to start from the same reference position (defined by the reference grating $\left.\left[N_{\text {ref }}=N_{\pi}, N_{v}=N_{0}\right]\right)$ when they are to display the varying grating $\left[N_{\text {ref }}=N_{\pi}, N_{v}=n\right]$. The sequence of alternated gratings is done for $n=$ $\{0 \ldots 255\}$ and for increasing frame rates. The resulting curves for the intensity of the first diffraction order are plotted in Fig. 8(b). From these results, it can be seen that a phase modulation range of $2 \pi$ can only be reached at frame rates lower than $63 \mathrm{~Hz}$. For frame rates of $505 \mathrm{~Hz}$ and higher, the maximum phase modulation range does not even reach $\pi$. In between, for the frame rates from 63 to $505 \mathrm{~Hz}$, the maximum phase modulation takes intermediate values between $\pi$ and $2 \pi$ [Fig. 7(b)].

To increase the phase modulation when the frequency increases, a new global LUT is computed with an extended BNS index level range of 128 gray levels corresponding to $[0,127]$ [Fig. 8(a)] from the experimental data obtained for the frame rate of $63 \mathrm{~Hz}$ (instead of the 88 gray levels used after the static calibration). Thus, for instance, a phase shift of $1.6 \pi$ corresponds to the index value 87 in this extended
BNS index level range [Fig. 8(a)], whereas it corresponded to $2 \pi$ in the former range for the static regime. A dynamic multi-LUT, based on the same method utilized for the static case, was also computed for a frequency of $63 \mathrm{~Hz}$ and is shown Fig. 8(b).

The global dynamic LUT is used to display new dynamic Ronchi gratings. The results are shown in Fig. $\underline{9}$, where it can be seen that, for all frequencies, the maximum phase modulation has increased.

\section{Experimental Results}

We have used the dynamic global- and multi-LUTs that were built for the frame rate of $63 \mathrm{~Hz}$ in several applications of dynamic DOE generation. Since the static maximum phase shift is just a little larger than $2 \pi, 63 \mathrm{~Hz}$ is the limit in practice. At this frame rate, all the 128 available BNS index levels are necessary to reach $2 \pi$. We tested the SLM at $205 \mathrm{~Hz}$, using the dynamic LUTs for a frame rate of $63 \mathrm{~Hz}$, to see if it would be possible to get still-acceptable results at a frame rate higher than $63 \mathrm{~Hz}$. We compared the experimental results obtained this way with those obtained using a static LUT. As an example of the dynamic phase modulation capabilities, we present the generation of a time variant Fresnel hologram. The holograms were computed using the iterative Fourier transform algorithm [29]. In our experiment, two holograms were alternately addressed to the SLM, operated with a display frame rate of $205 \mathrm{~Hz}$ : the UPC logo and a plain background. The camera was synchronized to capture the UPC logo, not the plain background. If the SLM response and the 


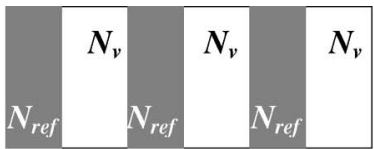

$\left(N_{\text {ref }}=128, N_{v}=0\right) \longleftrightarrow\left(N_{\text {ref }}=128, N_{v}=255\right)$

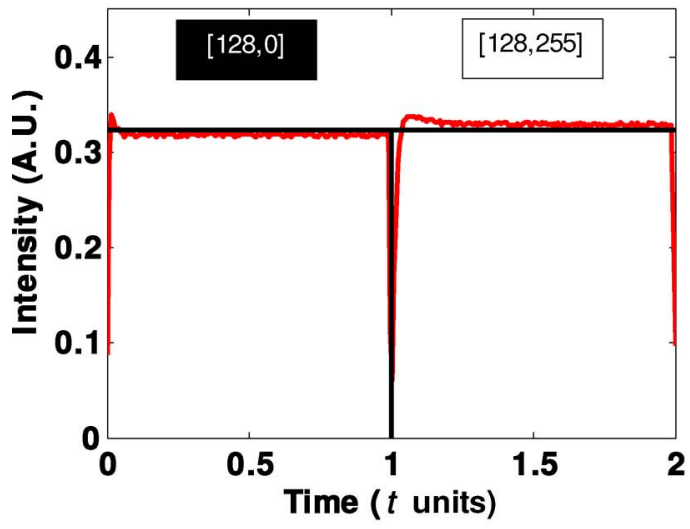

(a) $2 \mathrm{~Hz}$

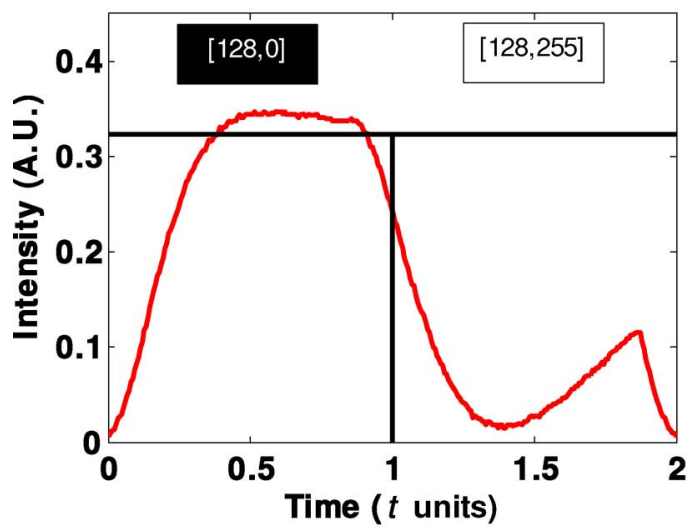

(c) $128 \mathrm{~Hz}$

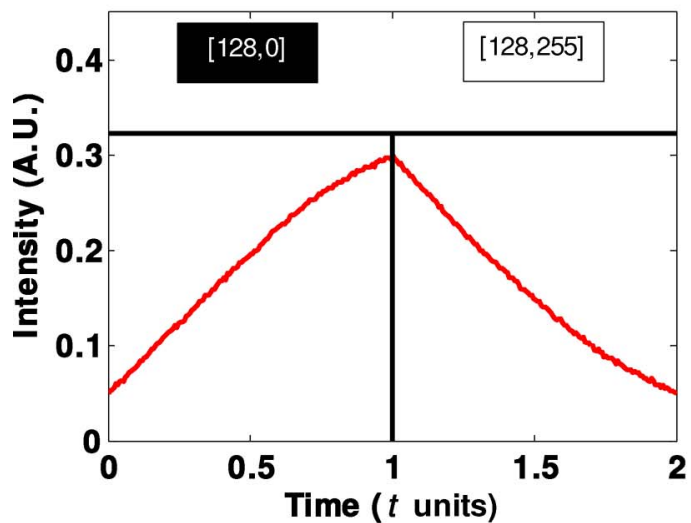

(e) $512 \mathrm{~Hz}$

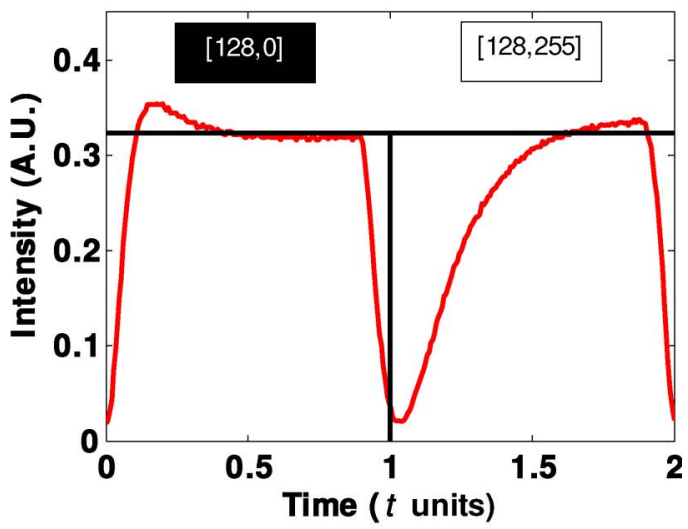

(b) $32 \mathrm{~Hz}$

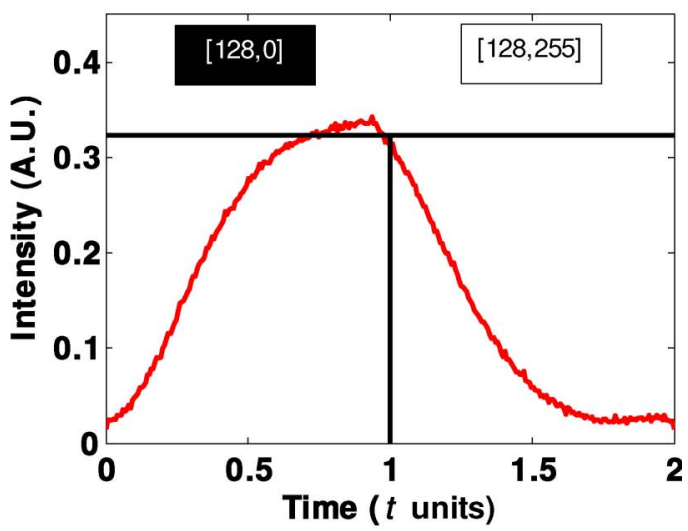

(d) $256 \mathrm{~Hz}$

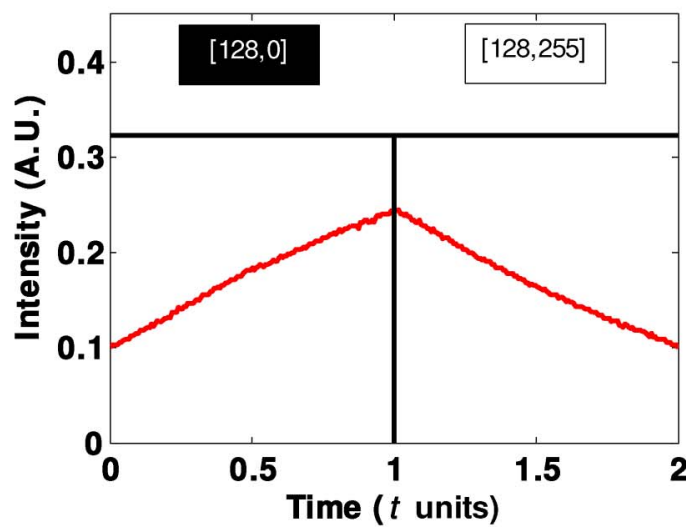

(f) $1011 \mathrm{~Hz}$

Fig. 6. (Color online) Intensities of the first diffraction order when two phase gratings are alternately addressed to the SLM for a time $t$ each at increasing rates (in comparison with the ideal T-shaped plot). The frame rate (in hertz) is equal to $1 / t$, with $t$ in seconds.

dynamic LUT worked correctly, then the camera would capture just the holographic image of the UPC logo, not the plain background. Otherwise, if some temporal disorder existed in the alternated dis- play of the holograms, then a noisy mixture of both reconstructed images would be registered, thus revealing the insufficient response of the device at the chosen frame rate. In Fig. 10(a) we have 
(a)
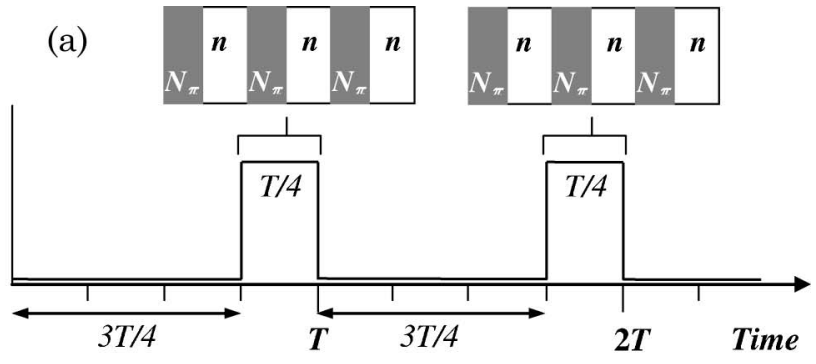

$\begin{array}{lllll}N_{\pi} & N_{0} & N_{0} \\ N_{\pi} & N_{\pi}\end{array}$
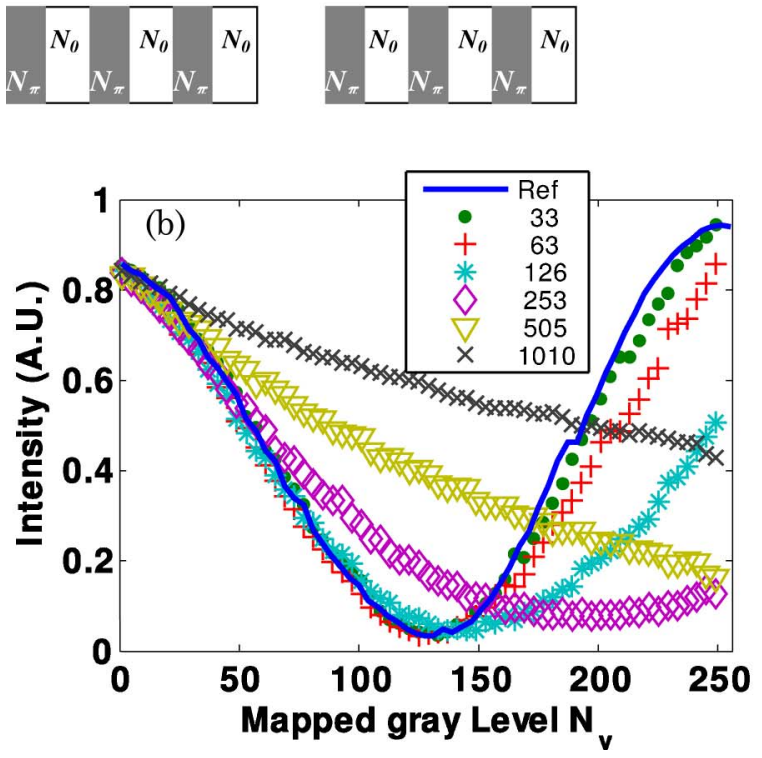

Fig. 7. (Color online) (a) Scheme of the temporal display of phase gratings for dynamic calibration, with $T$ the period, and $n=\{0 \ldots 255\}$. (b) Intensity curves for frame rates in the range of $[33,1010] \mathrm{Hz}$ when the variable gray level $N_{v}$ is interlaced with the reference gray level $\left(N_{\text {ref }}\right)$ using a LUT in the range $[0,87]$.

reproduced the time variant hologram using either the dynamic global-LUT or the multi-LUT of the BNS modulator and compared with the static multi-LUT. When the static multi-LUT is used, the modulator cannot display the two holograms with enough fidelity or correctly separated in time. As a consequence, a mixture of superposed images of poor quality is recorded by the camera. The dynamic LUTs, however, served to render sufficiently distinct the two holographic images. The camera was able to capture an improved UPC logo image with reduced noise caused by the presence of the other image (plain background). From the results obtained in Fig. 10(a), it is not possible to probe the advantages of using the dynamic multi-LUT instead of the dynamic globalLUT. The results obtained in both cases are similar. Since holograms are redundant, they are not so sensitive to the small encoding errors existing when a global-LUT is used instead of a multi-LUT. This does not happen, however, in the case of wavefront reconstruction, for which the use of multi-LUT is advantageous [19].

For the sake of comparison, the hologram of the UPC logo alone was addressed to the SLM for a steady display. The same three LUTs (one static and two


Fig. 8. (Color online) (a) Phase modulation used to generate a global dynamic LUT at $63 \mathrm{~Hz}$ and (b) dynamic multi-LUT value of the gray level giving a $2 \pi$ phase shift for the different regions of the SLM.

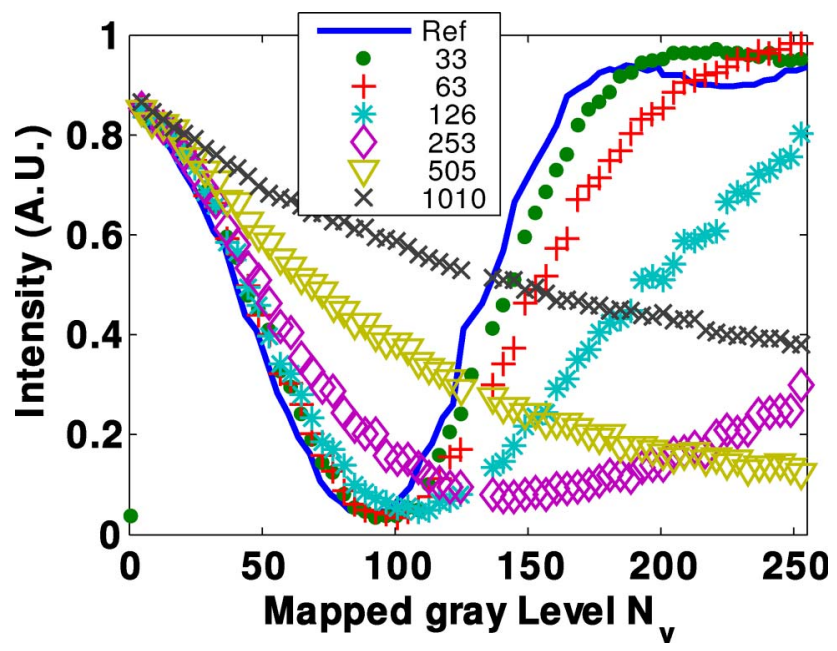

Fig. 9. (Color online) Intensity curves for frame rates in the range of $[33,1010] \mathrm{Hz}$ when the variable gray level $N_{v}$ is interlaced with reference gray level using an extended LUT [128, 255] (dynamic LUT). 


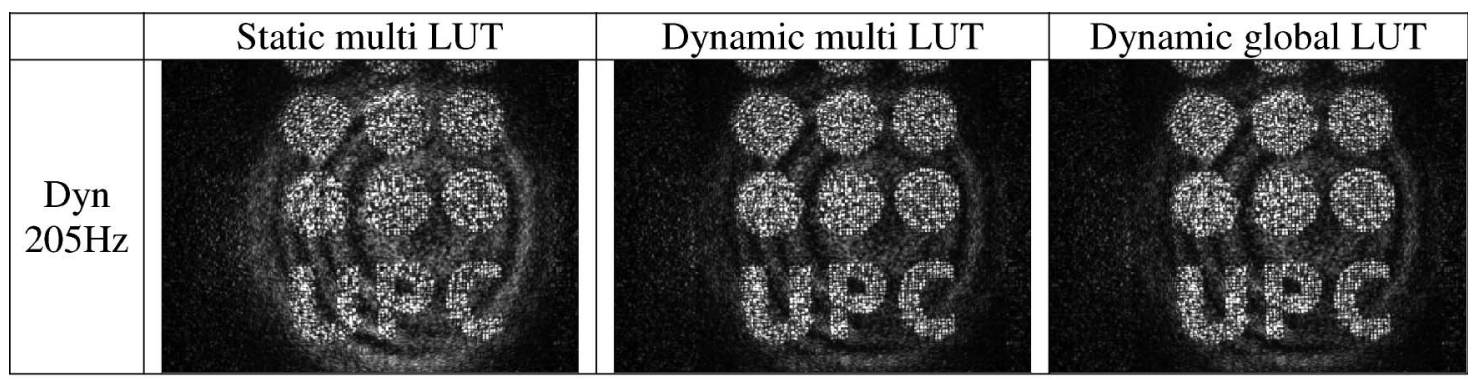

(a)

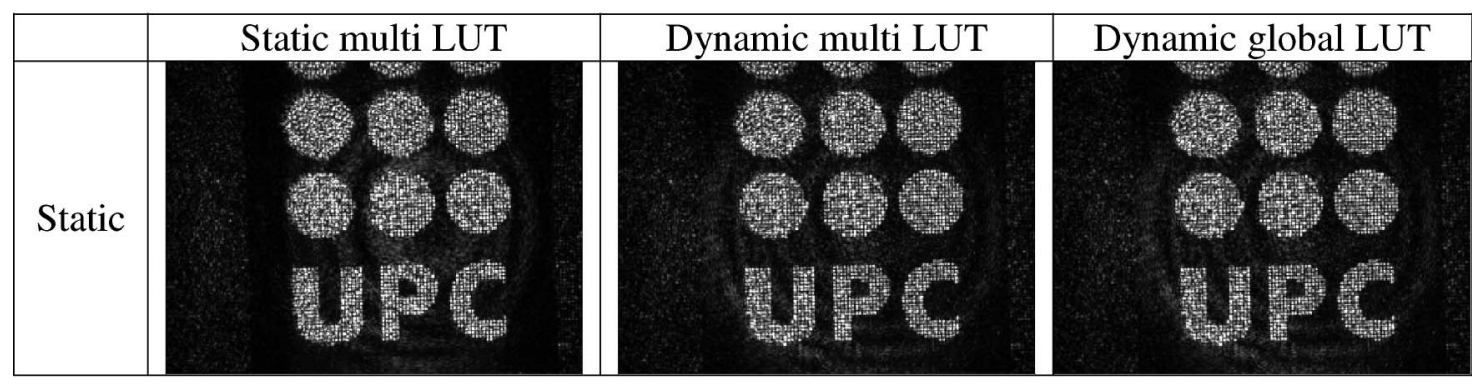

(b)

Fig. 10. (a) Optical reconstruction of a time-varying DOE: two holograms corresponding to the UPC logo and a plain background are alternatively addressed to the SLM. Different static and dynamic LUTs are used. (b) The same for a steady DOE (the hologram of the UPC logo alone).

dynamic) were separately used and the corresponding reconstructed image was recorded. Figure 10(b) shows the results, which are similar in terms of reconstruction quality.

\section{Conclusion}

For applications with high requirements of wavefront control, it is very important to experimentally characterize a reflective parallel-aligned LCoS SLM in terms of phase modulation versus the addressed gray level and inherent wavefront distortions. The pure phase-modulation capability is the principal advantage of these types of SLM. Their speed, however, which most of the time is limited to the video rate, is a strong limitation for their use in applications of optical processing. The BNS SLM we have used has a specific driver allowing a frequency range of up to $1011 \mathrm{~Hz}$ and, therefore, it was possible to complete the phase modulation characterization with a fullspeed characterization. This speed characterization showed that the SLM can operate at around $130 \mathrm{~Hz}$ and even faster for small phase changes. However, this frame rate reduces drastically to $32 \mathrm{~Hz}$ for extreme phase changes. We have proposed a method that allows an increase of the frame rate while maintaining a maximum phase modulation of $2 \pi$. Therefore, a new dynamic calibration has been carried out and the potential range of frame rates with maximum phase modulation of $2 \pi$ has been determined. The dynamic global-LUT and multi-LUT have been built for the frame rate of $63 \mathrm{~Hz}$, compatible with a $2 \pi$ phase stroke. We have displayed dynamic DOEs on the SLM at a frame rate of $205 \mathrm{~Hz}$ and the reconstructed patterns show an improvement of the recon- struction quality with the use of the dynamic LUT compared to the use of the static LUT.

The proposed method has its maximum efficiency if the maximum static phase modulation is really larger than $2 \pi$ and, in such a case, frame rates higher than $200 \mathrm{~Hz}$ could be achieved by using appropriate dynamic LUTs.

The authors are grateful to Laurent Bigué for many fruitful discussions. This research was partly supported by CNRS, Département Sciences et Technologies de l'Information (Equipe Projet Multi-Laboratoire fundings) and by Spanish Ministerio de Educación y Ciencia and Fondo Europeo de Desarrollo Regional funds (DPI2006-05479). Pierre Ambs was partly supported by the Rhenaphotonics interreg III European project.

\section{References}

1. Boulder Nonlinear Systems, Inc; Products (BNS, 2008), http:// www.bnonlinear.com.

2. Hamamatsu Spatial Light Modulator (Hamamatsu, 2008), http://www.hamamatsu.com.

3. Holoeye Photonics AG \& Holoeye Corporation (2008), http:// www.holoeye.com.

4. E. Frumker and Y. Silberberg, "Phase and amplitude pulse shaping with two-dimensional phase-only spatial light modulators," J. Opt. Soc. Am. B 24, 2940-2947 (2007).

5. A. Jesacher, C. Maurer, A. Schwaighofer, S. Bernet, and M. Ritsch-Marte, "Near-perfect hologram reconstruction with a spatial light modulator," Opt. Express 16, 2597-2603 (2008).

6. A. V. Kuzmenko and P. V. Yezhov, "Iterative algorithms for offaxis double-phase computer-generated holograms implemented with phase-only spatial light modulators," Appl. Opt. 46, 7392-7400 (2007). 
7. G. Milewski, D. Engstrom, and J. Bengtsson, "Diffractive optical elements designed for highly precise far-field generation in the presence of artifacts typical for pixelated spatial light modulators," Appl. Opt. 46, 95-105 (2007).

8. A. Jesacher, S. Fürhapter, S. Bernet, and M. Ritsch-Marte, "Diffractive optical tweezers in the Fresnel regime," Opt. Express 12, 2243-2250 (2004).

9. W. Osten, C. Kohler, and J. Liesener, "Evaluation and application of spatial light modulators for optical metrology," Opt. Pura Apl. 38, 71-81 (2005).

10. B. E. A. Saleh and K. Lu, "Theory and design of the liquid crystal TV as an optical spatial phase modulator," Opt. Eng. 29, 240-246 (1990).

11. C. Soutar and K. Lu, "Determination of the physical properties of an arbitrary twisted-nematic liquid crystal cell," Opt. Eng. 33, 2704-2712 (1994).

12. J. A. Davis, I. Moreno, and P. Tsai, "Polarization eigenstates for twisted-nematic liquid-crystal displays," Appl. Opt. 37, 937-945 (1998).

13. J. A. Davis, D. B. Allison, K. G. D’Nelly, M. L. Wilson, and I. Moreno, "Ambiguities in measuring the physical parameters for twisted-nematic liquid crystal spatial light modulators," Opt. Eng. 38, 705-709 (1999).

14. V. Duran, J. Lancis, E. Tajahuerce, and Z. Jaroszewicz, "Univocal determination of the cell parameters of a twisted nematic liquid crystal display by single-wavelength polarimetry," J. Appl. Phys. 97, 043101 (2005).

15. J. L. Harriman, A. Linnenberger, and S. A. Serati, "Improving spatial light modulator performance through phase compensation," Proc. SPIE 5553, 58-67 (2004).

16. X. Xun and R. W. Cohn, "Phase calibration of spatially nonuniform spatial light modulators," Appl. Opt. 43, 6400-6406 (2004).

17. M. Bock, S. K. Das, R. Grunwald, S. Osten, P. Staudt, and G. Stibenz, "Spectral and temporal response of liquidcrystal-on-silicon spatial light modulators," Appl. Phys. Lett. 92, 151105-151103 (2008).

18. N. W. Hart, A. Sergeyev, and T. J. Schulz, "Characterizing static aberrations in liquid crystal spatial light modulators using phase retrieval," Opt. Eng. 46, 086601 (2007).
19. J. Otón, P. Ambs, M. S. Millán, and E. Pérez-Cabré, "Multipoint phase calibration for improved compensation of inherent wavefront distortion in parallel aligned liquid crystal on silicon displays," Appl. Opt. 46, 5667-5679 (2007).

20. A. Jesacher, A. Schwaighofer, S. Fürhapter, C. Maurer, S. Bernet, and M. Ritsch-Marte, "Wavefront correction of spatial light modulators using an optical vortex image," Opt. Express 15, 5801-5808 (2007).

21. S. Serati, X. Xia, O. Mughal, and A. Linnenberger, "High-resolution phase-only spatial light modulators with submillisecond response," Proc. SPIE 5106, 138-145 (2003).

22. D. J. McKnight, K. M. Johnson, and R. A. Serati, " $256 \times 256$ liquid-crystal-on-silicon spatial light modulator," Appl. Opt. 33, 2775-2784 (1994).

23. T. Ewing, S. Serati, and K. Bauchert, "Optical correlator using four kilohertz analog spatial light modulator," Proc. SPIE 5437, 123-133 (2004).

24. D. J. Cho, S. T. Thurman, J. T. Donner, and G. M. Morris, "Characteristics of a $128 \times 128$ liquid-crystal spatial light modulator for wave-front generation," Opt. Lett. 23 (12), 969-971 (1998).

25. S. Serati and J. Harriman, "Spatial light modulators considerations for beam control in optical manipulation applications," Proc. SPIE 6326, 63262W (2006).

26. A. Lizana, I. Moreno, C. Iemmi, A. Márquez, J. Campos, and M. J. Yzuel, "Time-resolved Mueller matrix analysis of a liquid crystal on silicon display," Appl. Opt. 47, 4267-4274 (2008).

27. A. Lizana, I. Moreno, A. Márquez, C. Iemmi, E. Fernández, J. Campos, and M. J. Yzuel, "Time fluctuations of the phase modulation in a liquid crystal on silicon display: characterization and effects in diffractive optics," Opt. Express 16, 16711-16722 (2008).

28. Z. Zhang, G. Lu, and F. T. S. Yu, "Simple method for measuring phase modulation in liquid crystal televisions," Opt. Eng. 33, 3018-3022 (1994).

29. F. Wyrowski and O. Bryngdahl, "Iterative Fourier-transform algorithm applied to computer holography," J. Opt. Soc. Am. A 5, 1058-1065 (1988). 